\begin{tabular}{|c|l|}
\hline Title & $\begin{array}{l}\text { What is Degussa (Evonik) P25? Crystall line composition analy sis, reconstruction from isolated pure particles and } \\
\text { photocatalytic activity test }\end{array}$ \\
\hline Author(s) & Ohtani, B.; Prieto-Mahaney, 0. O.; Li, D.; A be, R. \\
\hline Citation & $\begin{array}{l}\text { Journal of Photochemistry and Photobiology A : Chemistry, 216(2-3), 179-182 } \\
\text { https:/doi.org/10.1016/.jphotochem.2010.07.024 }\end{array}$ \\
\hline Issue Date & 2010-12-15 \\
\hline Doc URL & http://hdl.handle.net/2115/44837 \\
\hline Type & article (author version) \\
\hline File Information & JPPA 216-2-3_179-182.pdf \\
\hline
\end{tabular}

Instructions for use 


\title{
What is Degussa (Evonik) P25? Crystalline composition analysis, reconstruction from isolated pure particles and photocatalytic activity test
}

\author{
B. Ohtani, * O. O. Prieto-Mahaney, D. Li, R. Abe \\ Catalysis Research Center, Hokkaido University, Sapporo 001-0021, Japan
}

\begin{abstract}
Anatase and rutile crystallites were isolated from Degussa (Evonik) P25 by selective dissolution with a hydrogen peroxide-ammonia mixture and diluted hydrofluoric acid, respectively, and used as standard samples for calibration curves of X-ray diffraction analyses. The results showed that P25 contains more than 70\% anatase with a minor amount of rutile and a small amount of amorphous phase. The composition anatase/rutile/amorphous could be determined by analysis of P25 mixed with an internal standard, nickel(II) oxide. However, it was also found that the composition of P25 used in this study was inhomogeneous and changed depending on the position of sampling from the same package. Comparison of activities of original P25 and reconstructed P25 with those of isolated anatase and rutile particles suggested a less probable synergetic effect of the co-presence of anatase and rutile.
\end{abstract}

Keywords: powder X-ray diffraction; anatase-rutile-amorphous mixture: inhomogeneity of crystalline composition; less-probable synergetic effect

\section{Introduction}

Degussa (Evonik) P25, Aeroxide $\mathrm{TiO}_{2} \mathrm{P} 25$, is a titania photocatalyst that is used widely because of its relatively high levels of activity in many photocatalytic reaction systems.

\footnotetext{
*Corresponding Author. Tel.: +81 11706 9132; fax: +81 11706 9133. E-mail address: ohtani@cat.hokudai.ac.jp (B. Ohtani).
} 
Actually, it is not easy to find a photocatalyst showing activity higher than that of P25, and P25 has therefore been used as a de-facto standard titania photocatalyst; photocatalytic reactions of P25 have been reported in more than thousand papers since 1990 [1]. It is well known that P25 is composed of anatase and rutile crystallites, the reported ratio being typically 70:30 or 80:20, but it seems that nobody knows the exact crystalline composition [2], presumably due to a lack of methodology for determination of crystalline contents in nanometer-sized particulate samples [3]. Another myth, a hypothesis not proved scientifically, regarding P25 is that the co-presence of anatase and rutile crystallites induces the high level of photocatalytic activity; transfer of photoexcited electrons and positive holes between interconnecting anatase and rutile particles may enhance charge separation and hence improve the efficiency of utilization of electron-hole pairs [4]. However, as far as the authors know, there have been no reports showing enhancement of activity compared with pure anatase and rutile parts of P25 or with the anatase and rutile particles without any electrical contact: no data have been shown for expected lower activity of pure anatase and rutile particles alone. Thus, despite the long history of photocatalysis studies, it seems that we have not yet known what P25 is from the standpoints of structural and photocatalytic characteristics.

In a scientific sense, isolation of anatase and rutile crystallites from P25 is necessary to determine the crystalline composition and to check the synergetic effect of anatase and rutile. Isolation of rutile by selective dissolution of anatase from P25 has been reported by Ohno et al. [5]. Recently, we have developed a method to isolate anatase by preferable dissolution of rutile from P25 using a mixed solution of hydrogen peroxide and ammonia [6]. Our attempts to determine the crystalline composition and to check the synergetic effect of an anatase-rutile mixture using isolated anatase and rutile powder as standards are reported in this paper.

\section{Experimental}

\subsection{Materials}

The sample P25 was supplied by Nippon Aerosil in a scale of $1 \mathrm{~kg}$. Anatase [6] and 
rutile [5] crystallites were isolated according to previous reports. It has been reported that P25 contains an amorphous phase based on transmission electron microscopy (TEM) $[7,8]$, but there have been no attempts to isolate the amorphous phase from P25. For reconstruction of P25 by mixing pure anatase, rutile and amorphous particles, a commercial sample of amorphous titania (Wako Pure Chemical) was used as well as the isolated anatase and rutile.

\subsection{Characterization of titania samples}

XRD patterns of samples were recorded on a Rigaku RINT ULTIMA powder X-ray diffractometer using copper $\mathrm{K}_{\alpha}$ radiation $(40 \mathrm{kV}, 30 \mathrm{~mA})$ with a scanning rate of 1 degree $\min ^{-1}$ (0.010-degree step). Peak intensity was measured by integration of a given peak, 101 (25.3 degrees) and 110 (27.4 degrees) for anatase and rutile, respectively, after baseline correction. Calibration curves for anatase and rutile were obtained by XRD analyses of samples of a mixture of isolated anatase and rutile powders with various ratios containing a 20wt\% internal standard, nickel(II) oxide (NiO; Wako Pure Chemical). Procedures for other characterizations of titania samples were reported previously [6].

\subsection{Photocatalytic reaction}

Four test photocatalytic reactions were as follows: (a) oxidative decomposition of acetic acid in an aerated 5vol\% aqueous solution, (b) oxidative decomposition of acetaldehyde (ca. 2200 ppm) in air, (c) dehydrogenation of methanol in a deaerated 50vol\% aqueous solution containing hydrogen hexachloroplatinate(IV) $\left(\mathrm{H}_{2} \mathrm{PtCl}_{6} \cdot 6 \mathrm{H}_{2} \mathrm{O}\right)$ for in-situ platinum deposition (2wt\%) and (d) oxygen $\left(\mathrm{O}_{2}\right)$ liberation from a deaerated aqueous silver sulfate solution (25 mmol $\mathrm{L}^{-1}$ ) along with deposition of silver metal on the surface of the photocatalyst. A 400-W mercury arc (Eiko-sha 400; ca. $25 \mathrm{~mW} \mathrm{~cm}^{-2}$ at 300-400 nm) was used for irradiation at $>300 \mathrm{~nm}$ except for reaction (b), in which a 300-W xenon arc (ILC Technology CERMAX-LX300F; ca. $50 \mathrm{~mW} \mathrm{~cm}^{-2}$ at 300-400 nm) was used. The experimental details were reported elsewhere: reactions (a) — (c) $[9,10]$ and reaction (d) [11]. 


\section{Results and Discussion}

\subsection{Crystalline composition analyses}

Figure 1 shows parts of normalized X-ray diffraction (XRD) patterns of isolated pure anatase and rutile powders and original P25. Contamination of rutile in anatase was not detected, and vice versa. It should be noted that the widths of peaks at ca. 25.3 and 27.4 degrees for anatase and rutile, respectively, of isolated and original samples were practically the same. Since the width of an XRD peak is a measure of crystallite size (correctly speaking, depth of a crystallite measured in the direction vertical to the lattice plane giving the diffraction peak $[3,12])$, the results suggest that anatase and rutile crystallites were isolated without changing their size. Both the employed isolation procedures for anatase and rutile involved dissolution, and the relatively low isolation yields suggest that both crystallites were dissolved but that the small difference in the rate of dissolution induced negligible contamination. A possible reason for negligible change in crystallite size even under these conditions in which smaller particles may be dissolved faster is that the sizes of anatase and rutile crystallites in original P25 are narrowly distributed.

Figure 2 shows the calibration curves for anatase and rutile crystallites in P25. Linearity was observed for both plots as expected. Similar linearity was reported for the first time by Spurr and Myers [13] plots between weight and XRD peak intensity ratios of artificial anatase-rutile mixtures, and calibration curves reported therein have been frequently used to determine the anatase-rutile ratio in titania powders, neglecting the possible presence of amorphous phase. Use of an internal standard, such as $\mathrm{NiO}$ in this study, enables the determination of absolute content of anatase or rutile if pure anatase or rutile samples are obtained. However, we always face a problem that relative intensity of the XRD peak of a standard changes depending on the kind of standard [14,15]. (Although Jensen et al. claimed that "theoretical intensity ratio" could be obtained and that P25 consisted of 73\% anatase and $18 \%$ rutile [16], there was no interpretation on applicability of obtained calibration curves to fine titania particles such as P25.) There are at least two possible reasons for this fact. One is possible existence of an amorphous phase, giving no XRD peaks, and the other is that XRD peak intensity may depend on the size of samples. The latter has been supported by the 
results of comparison of amorphous contents evaluated by thermal and XRD analyses [17]; it was suggested that anatase particles of crystallites that are smaller than $30 \mathrm{~nm}$ might give less intense peaks. Therefore, it is necessary to make calibration curves using pure anatase and rutile crystallites contained in their original mixture for the determination of crystalline composition. Now, such ideal calibration curves have been obtained for P25.

Typical crystalline composition of P25 was evaluated to be $78 \%$ anatase and $14 \%$ rutile. Assuming the remaining $8 \%$ part corresponds to amorphous phase, the anatase-rutile-amorphous ratio is determined to be 78:14:8. This is the first example, as far as we know, of determination of crystalline composition for a sample which is originally a mixture, not an artificial mixture using pure crystalline phases extracted from the sample. However, during studies on reproducibility of the present crystalline composition analyses, we found that the ratio changed from time to time as shown in Table 1; it was sometimes observed that there was practically no amorphous phase even if a portion of the sample powder was collected from the same package. (Jensen's group reported similar fluctuations of crystalline composition of P25 using their method in different publications $[16,18]$, but no relating descriptions were found.) Such fluctuation is attributable to inhomogeneity of the crystalline composition of P25. Ohno et al. observed anatase, rutile and amorphous domains in TEM images of as-supplied P25 [8], presumably because P25 is prepared by gas-phase flame synthesis; anatase, rutile and amorphous may be produced depending on the flame conditions. The inhomogeneity of as-supplied P25 in the same package was also supported by the fact that P25, which was suspended in water and then dried up, gave small fluctuation in crystalline composition (with appreciable decrease in photocatalytic activity the extent of which depends on the kind of reaction). Datye et al. claimed that P25 does not contain a detectable amount of amorphous phase [19] in contrast to the report [8] by Ohno's group as described above. This discrepancy can be interpreted by the inhomogeneity, in the same package and among different batches of production, of the crystalline composition of P25. Also suggested in Table 1 is that specific interaction between anatase and rutile crystallites and coverage of anatase and rutile particles with amorphous layers, as suggested previously [7], seem less probable, being consistent with the claims by Datye et al. [19] and Ohno et al. 
[8], since the ratio of anatase to rutile fluctuated appreciably and the content of amorphous changed independently.

The results described above suggest that P25 is a simple mixture of anatase (major), rutile and amorphous (minor) titania with different ratios depending on the sample production batch and position in a package and that descriptions of the crystalline composition, if determined by a scientifically reasonable method, does not make sense; the observed crystalline composition, though appreciably fluctuated, reported here cannot be applied to P25 stored in other laboratories.

\subsection{Reconstruction of $\mathrm{P} 25$ and photocatalytic activity test}

Anatase and rutile single phase particles could be isolated from P25 with negligible change in particle size, while there have been found no ways for extraction of amorphous phase. In the following studies on reconstruction of P25 and photocatalytic activity test, commercial amorphous titania was used as an amorphous component. Since, as has been reported previously, photocatalytic activity of amorphous titania seemed negligible [17] and the content of amorphous phase was relatively small, the influence of using different types of amorphous particles is expected to be negligible.

Figure 3 shows the normalized photocatalytic activity of original P25, isolated pure anatase, isolated pure rutile and reconstructed P25 (R-P25), i.e, mixture of isolated pure anatase and rutile particles with amorphous powder in the ratio of 78:14:8, which was the same as that for original P25 used in this comparison. The liquid-phase process of isolation of anatase and rutile from P25 included the steps of washing with water, ultrasonication and drying at ca. $120{ }^{\circ} \mathrm{C}$ in air. Preliminary experiments revealed that the photocatalytic activity of as-received P25 was decreased (5-30\% depending on the kind of reaction) by similar wet processes, though the reason is not clear at present. Therefore original P25 that was treated in a similar way was used, and its photocatalytic activity, i.e., reaction rate, was normalized to $100 \%$ in each reaction system.

First, R-P25 showed activity similar to that of P25 in the four kinds of photocatalytic reaction systems; the difference was $-11 \sim+19 \%$. These facts suggest that there was 
negligible effect of change in surface structures on the activities even if any changes were introduced by the isolation processes. It should be noted that R-P25 was a simple mixture without any treatment; three kinds of particles were weighed in a reaction tube and a reaction solution was poured in the tube. The comparable photocatalytic activities of R-P25 also suggested the absence of strong interaction among the particles or negligible influence of such interparticle interaction, if present, on photocatalytic activity. Thus, it seems that anatase, rutile and amorphous phases in P25 behave independently when working as a photocatalyst. This is also supported by the fact that the weighted sum of photocatalytic activities calculated from those of isolated pure anatase, rutile and amorphous particles was also similar to the normalized photocatalytic activity of P25 and R-P25 in each reaction system, as shown in Fig. 3 (Relatively large difference in reaction (d), oxygen evolution, is attributable to error since the content of highly active pure rutile was very small.).

Second, except for reaction (c), pure anatase (reactions (a) and (b)) or pure rutile (reaction (d)) showed activity higher than those of P25 and R-P25, indicating that photocatalytic activity can be improved by isolation of active crystalline phase, anatase or rutile, depending on the kind of reaction. In other words, the results shown in Fig. 3 suggest the absence of a synergetic effect of the co-presence of anatase and rutile in P25 on photocatalytic activity. Ohno et al. reported that an artificial mixture of anatase and rutile crystallites had better photocatalytic activity than that of pure anatase and rutile alone [20] based on a plot of photocatalytic activity for oxidation of naphthalene as a function of anatase (rutile) content with a curve that is concave upward, i.e., optimum activity was observed with a $30 \%$ anatase sample. Figure 4 shows the plot of photocatalytic activity observed in reaction (a) as a function of anatase content in anatase-rutile binary mixtures without amorphous phase. It is obvious from the linear plots that an artificial mixture of isolated pure anatase and rutile crystallites shows no synergetic effect. Furthermore, a plot of the activity of original (water-treated) P25 was close to the linear line for artificial mixtures, being consistent with the above-mentioned hypothesis of negligible synergetic effect.

On the other hand, in reaction (c), the photocatalytic activities of P25 and R-P25 were better than those of isolated pure anatase and rutile particles, and the weighted sum of 
normalized activities of pure anatase, rutile and amorphous phases was 74 (Figure 3). Although these results might suggest a synergetic effect of the co-presense of anatase and rutile in P25 and R-P25, we doubt the reality of the synergism, since this photocatalytic reaction requires platinum loading onto a photocatalyst. It has been shown that only photocatalyst particles with platinum deposits in appropriate amount can be active [21] and that rutile titania shows activities comparable to those of anatase titania [11,22]. Therefore, distribution and size of platinum deposits should be the same on all photocatalysts, while it is actually impossible to completely control the platinum deposition. Analysis of the distribution and size of platinum deposits on P25 and reproduction of R-P25 with the same platinum deposits are necessary for proof of the presence or absence of synergism. Studies along this line are now in progress.

\section{Conclusions}

Studies on structural and photocatalytic properties of P25 using isolated pure anatase and rutile particles as a reference revealed that components of P25, anatase, rutile and amorphous phases behave independently without any interactions. This structural characteristic of P25 has been suggested by Detye et al. based on results of TEM studies [19]. Core-shell type or interconnected anatase-rutile composite structure and synergetic effect of the co-presence of anatase and rutile on photocatalytic activity of P25, both of which have been widely believed in the field of photocatalysis, are suggested to be myths, speculations without any scientific proof. Another important finding is that photocatalytic activity of fine titania particles such as P25 and the isolated anatase and rutile particles changes appreciably by a wet process, e.g., drying in air of paste with water, presumably due to aggregation of particles by inter-particle dehydration. S Studies on this problem are currently underway.

\section{Acknowledgments}

Sample P25 was a gift from Nippon Aerosil Co. Ltd. This work was partly supported by a Grant-in-Aid for Scientific Research (A) from the Ministry of Education, Culture, Sports, Science and Technology (MEXT) of the Japanese Government and by Project 
to Create Photocatalyst Industry for Recycling-oriented Society supported by NEDO, New Energy and Industrial Technology Development Organization. We thank Professor Wataru Ueda (Catalysis Research Center, Hokkaido University) for permission to use an X-ray diffractometer.

\section{References}

[1] ISI Web of Knowledge, Thomson Reuters.

[2] The supplier, Degussa (Evonik) does not report the crystalline composition of P25. Many papers claiming the crystalline composition referred to papers which had not shown the method of determination.

[3] B. Ohtani, Preparing Articles on Photocatalysis-Beyond The Illusions, Misconceptions and Speculation, Chem. Lett., 37 (2008) 216.

[4] D. C. Hurum, A. G. Agrios, K. A. Gray, T. Rajh, C. Thurnauer, Explaining the Enhanced Photocatalytic Activity of Degussa P25 Mixed-Phase $\mathrm{TiO}_{2}$ Using EPR, J. Phys. Chem. B 107 (2003) 4545.

[5] T. Ohno, K. Sarukawa, M. Matsumura, Photocatalytic Activities of Pure Rutile Particles Isolated from $\mathrm{TiO}_{2}$ Powder by Dissolving the Anatase Component in HF Solution, J. Phys. Chem. B 105 (2001) 2417.

[6] B. Ohtani, Y. Azuma, D. Li, T. Ihara, R. Abe, Isolation of Anatase Crystallites from Anatase-Rutile Mixed Particles by Dissolution with Hydrogen Peroxide and Ammonia, Trans. Mater. Res. Soc. Jpn., 32 (2007) 401.

[7] R. I. Bickley, T. Gonzalez-Carreno, J. S. Lees, L. Palmisano, R. J. D. Tilley, A Structural Investigation of Titanium Dioxide Photocatalysts, J. Solid State Chem. 92 (1991) 178.

[8] T. Ohno, K. Sarukawa, K. Tokieda, M. Matsumura, Morphology of a $\mathrm{TiO}_{2}$ photocatalyst (Degussa, P-25) consisting of anatase and rutile crystalline phases, J. Catal. 203 (2001) 82.

[9] F. Amano, O.O. Prieto-Mahaney, Y. Terada, T. Yasumoto, T. Shibayama, B. Ohtani, 
Decahedral Single-Crystalline Particles of Anatase Titanium(IV) Oxide with High Photocatalytic Activity, Chem. Mater. 21 (2009) 2601.

[10] F. Amano, T. Yasumoto, O.O. Prieto-Mahaney, S. Uchida, T. Shibayama, B. Ohtani, Photocatalytic Activity of Octahedral Single-Crystalline Mesoparticles of Anatase Titanium(IV) Oxide, Chem. Commun 45 (2009) 2311.

[11] O. O. Prieto-Mahaney, N. Murakami, R. Abe, B. Ohtani, Correlation between Photocatalytic Activities and Structural and Physical Properties of Titanium(IV) Oxide Powders, Chem. Lett. 38 (2009) 238.

[12] H. P. Klug and L. E. Alexander, X-Ray diffraction procedures for polycrystalline and amorphous materials (2nd edition), Wiley, New York (1974) p. 687.

[13] R. A. Spurr, H. Myers, Quantitative Analysis of Anatase-Rutile Mixtures with an X-ray Diffractometer, Anal. Chem. 29 (1957) 760.

[14] S.-i. Nishimoto, B. Ohtani, A. Sakamoto, T. Kagiya, Photocatalytic Activities of Titanium(IV) Oxide Prepared from Titanium(IV) Sulfate, Nippon Kagaku Kaishi, (1984) 246.

[15] S.-i. Nishimoto, B. Ohtani, H. Kajiwara, T. Kagiya, Correlation of the Crystal Structure of Titanium Dioxide Prepared from Titanium Tetra-2-propoxide with the Photocatalytic Activity for Redox Reactions in Aqueous Propan-2-ol and Silver Salt Solutions, J. Chem. Soc., Faraday Trans. 1, 81 (1985) 61.

[16] H. Jensen, K. D. Joensen, J.-E. Jorgensen, J. S. Pedersen, E. G. Sogaard, Characterization of nanosized partly crystalline photocatalysts, J. Nanoparticle Res. 6 (2004) 519.

[17] B. Ohtani, Y. Ogawa, S.-i. Nishimoto, Photocatalytic Activity of Amorphous-Anatase Mixture of Titanium(IV) Oxide Particles Suspended in Aqueous Solutions, J. Phys. Chem. B 101 (1997) 3746.

[18] M. E. Simonsen, H. Jensen, Z. Li, E. G. Sogaard, Surface properties and photocatalytic activity of nanocrystalline titania films, J. Photochem. Photobiol A: Chem. 200 (2008) 192. 
[19] A. K. Datye, G. Riegel, J. R. Bolton, M. Huang, M. R. Prairie, Microstructural Characterization of a Fumed Titanium Dioxide Photocatalyst, J. Solid State Chem. 115 (1995) 236.

[20] T. Ohno, K. Tokieda, S. Higashida, M. Matsumura, Synergism between rutile and anatase $\mathrm{TiO}_{2}$ particles in photocatalytic oxidation of naphthalene, Appl. Catal. A: Gen. 244 (2003) 383.

[21] B. Ohtani, K. Iwai, S.-i. Nishimoto, S. Sato, Role of Platinum Deposits on Titanium(IV) Oxide Particles: Structural and Kinetic Analyses of Photocatalytic Reaction in Aqueous Alcohol and Amino Acid Solutions, J. Phys. Chem. B. 101 (1997) 3349.

[22] T. Torimoto, N. Nakamura, S. Ikeda, B. Ohtani, Discrimination of Active Crystalline Phase in Anatase-Rutile Mixed Titanium(IV) Oxide Photocatalysts through Action Spectrum Analyses, Phys. Chem. Chem. Phys. 4 (2002) 5910. 
Table 1 Crystalline composition of P25 collected from the same package.

\begin{tabular}{ccccc}
\hline \multirow{2}{*}{ entry } & \multicolumn{3}{c}{ \%composition } & anatase/rutile ratio \\
\cline { 2 - 4 } & anatase & rutile & amorphous & 5.6 \\
\hline 1 & 78 & 14 & 8 & 5.2 \\
2 & 73 & 14 & 13 & 5.1 \\
3 & 82 & 16 & 2 & 4.9 \\
4 & 83 & 17 & 0 & 5.3 \\
5 & 84 & 16 & 0 & 5.7 \\
6 & 85 & 15 & 0 & \\
\hline
\end{tabular}

\section{Figure captions}

Fig. 1 Parts of normalized X-ray diffraction (XRD) patterns of isolated anatase (solid line) and rutile (dotted line) powders and original P25 (white). Patterns of pure anatase and rutile were normalized.

Fig. 2 Calibration curves of anatase and rutile obtained using a mixture of isolated pure anatase and rutile with a given amount (20wt\%) of $\mathrm{NiO}$ as an internal standard. Squared correlation coefficients of anatase and rutile were 0.9991 and 0.9989 , respectively.

Fig. 3 Normalized photocatalytic activities of (from left to right) original P25, isolated anatase and rutile, commercial amorphous titania and R-P25 reconstructed from isolated anatase and rutile with amorphous titania in (a) oxidative decomposition of acetic acid in an aerated aqueous solution, (b) oxidative decomposition of acetaldehyde in air, (c) dehydrogenation of methanol in a deaerated aqueous solution and (d) oxygen liberation from a deaerated aqueous silver sulfate solution. Figures on the data of R-P25 show weighted sum of photocatalytic activities calculated from those of isolated pure anatase, rutile and amorphous particles. 
Fig. 4 Photocatalytic activities of artificial binary mixtures of anatase and rutile without amorphous phase as a function of anatase (rutile) content for reaction (a). The average activities of original P25 samples are also plotted; since the exact crystalline composition was not determined for P25 samples, the horizontal error bar shows probable range of anatase content in anatase-rutile mixture, neglecting an amorphous phase. 
Fig. 1

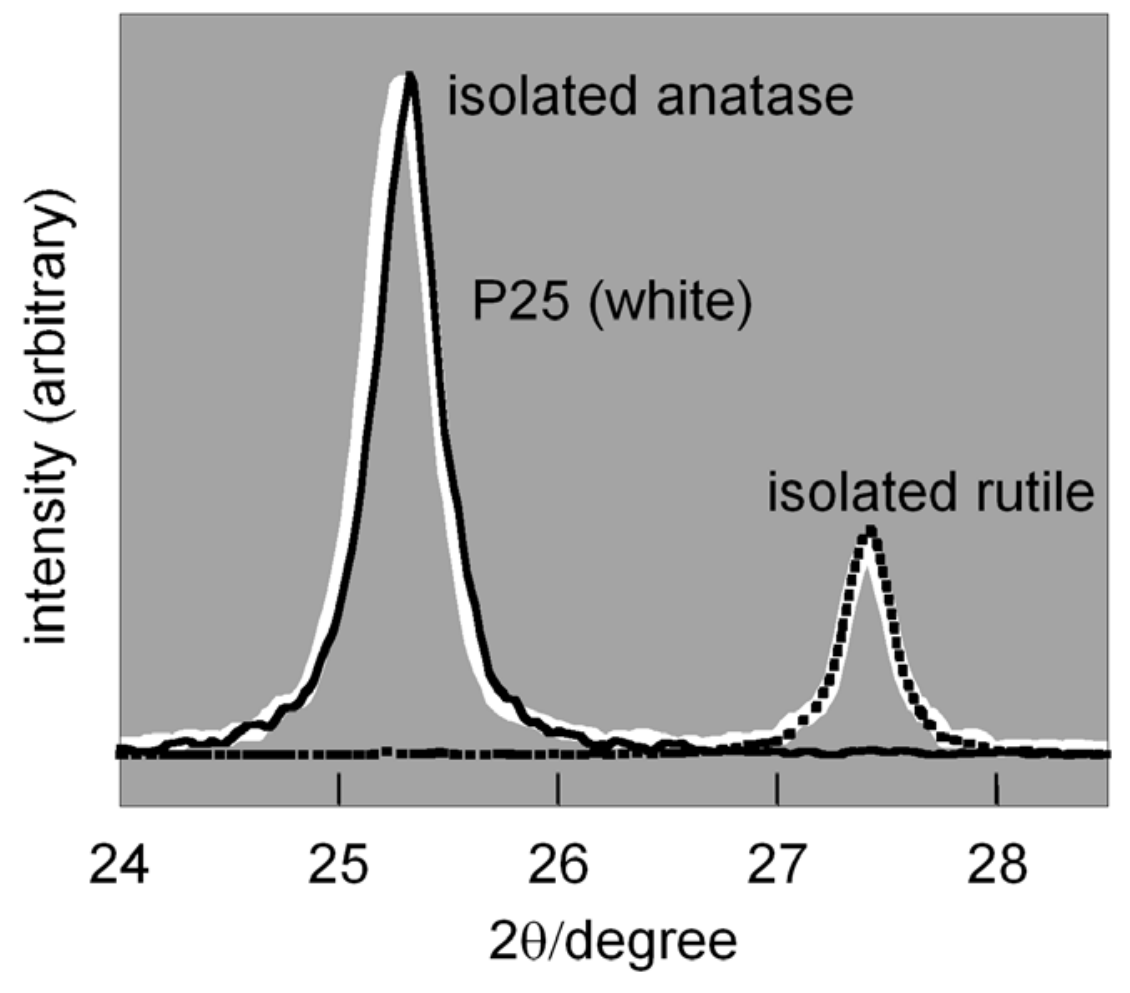


Fig. 2

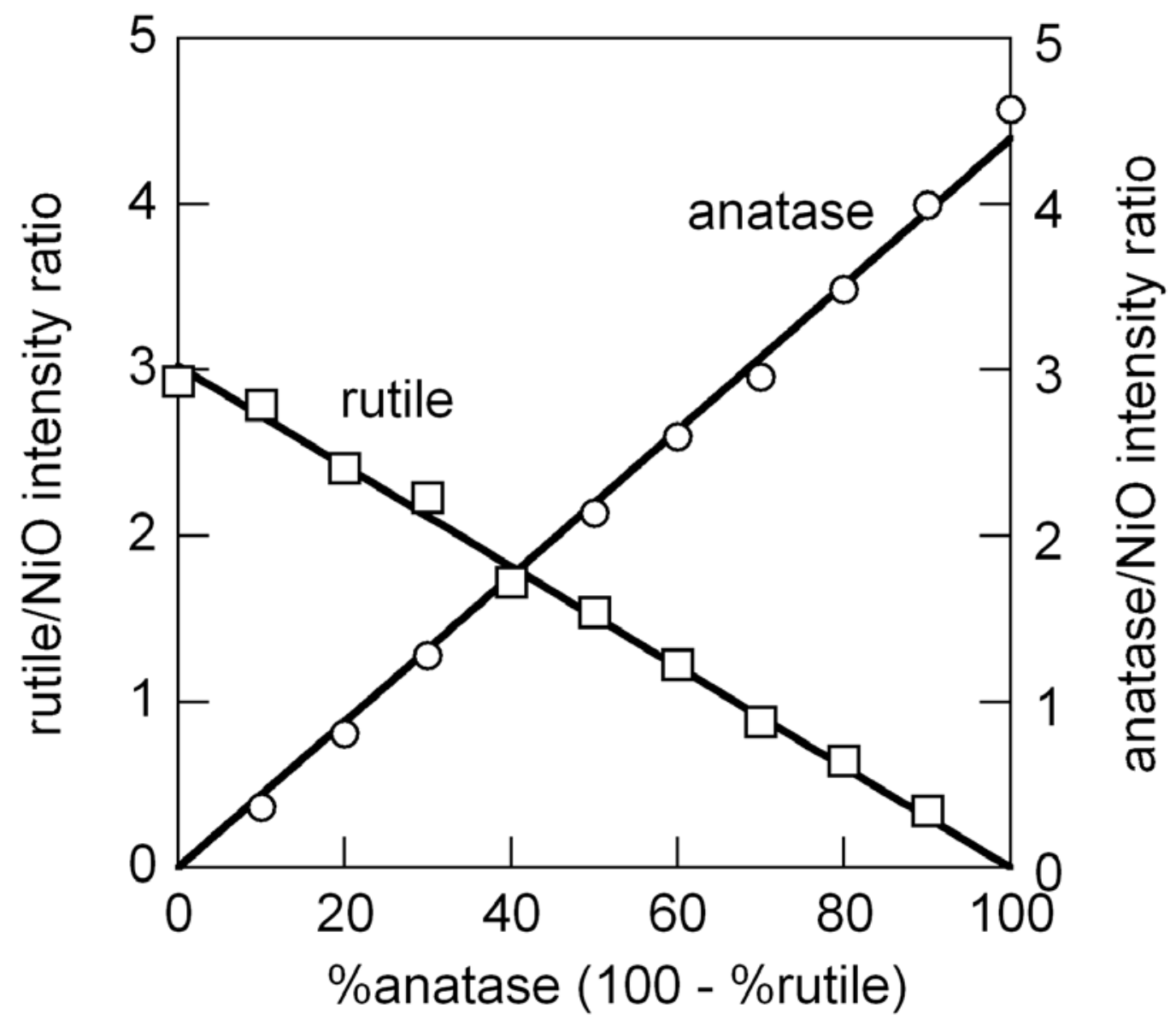


Fig. 3

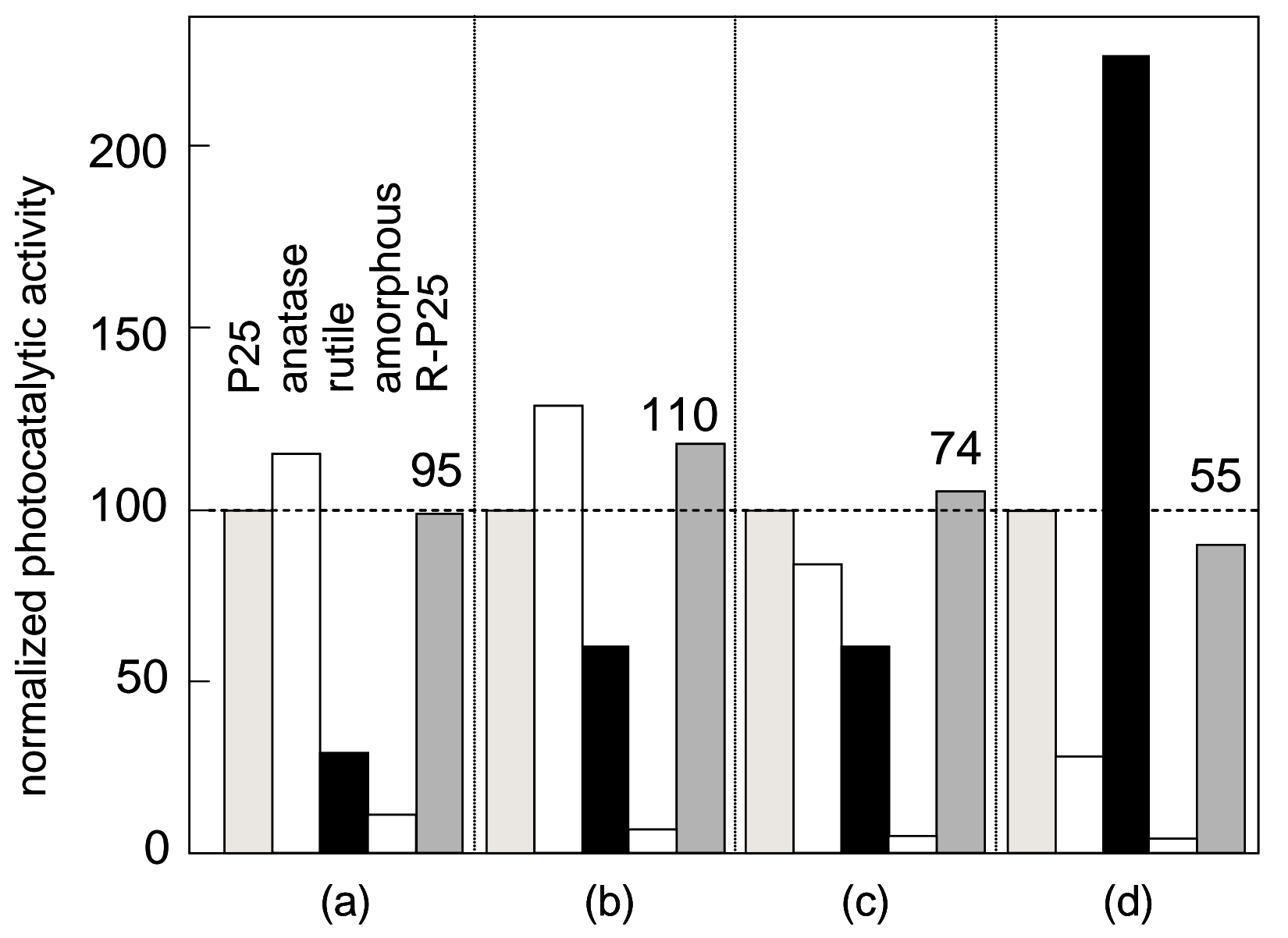


Fig. 4

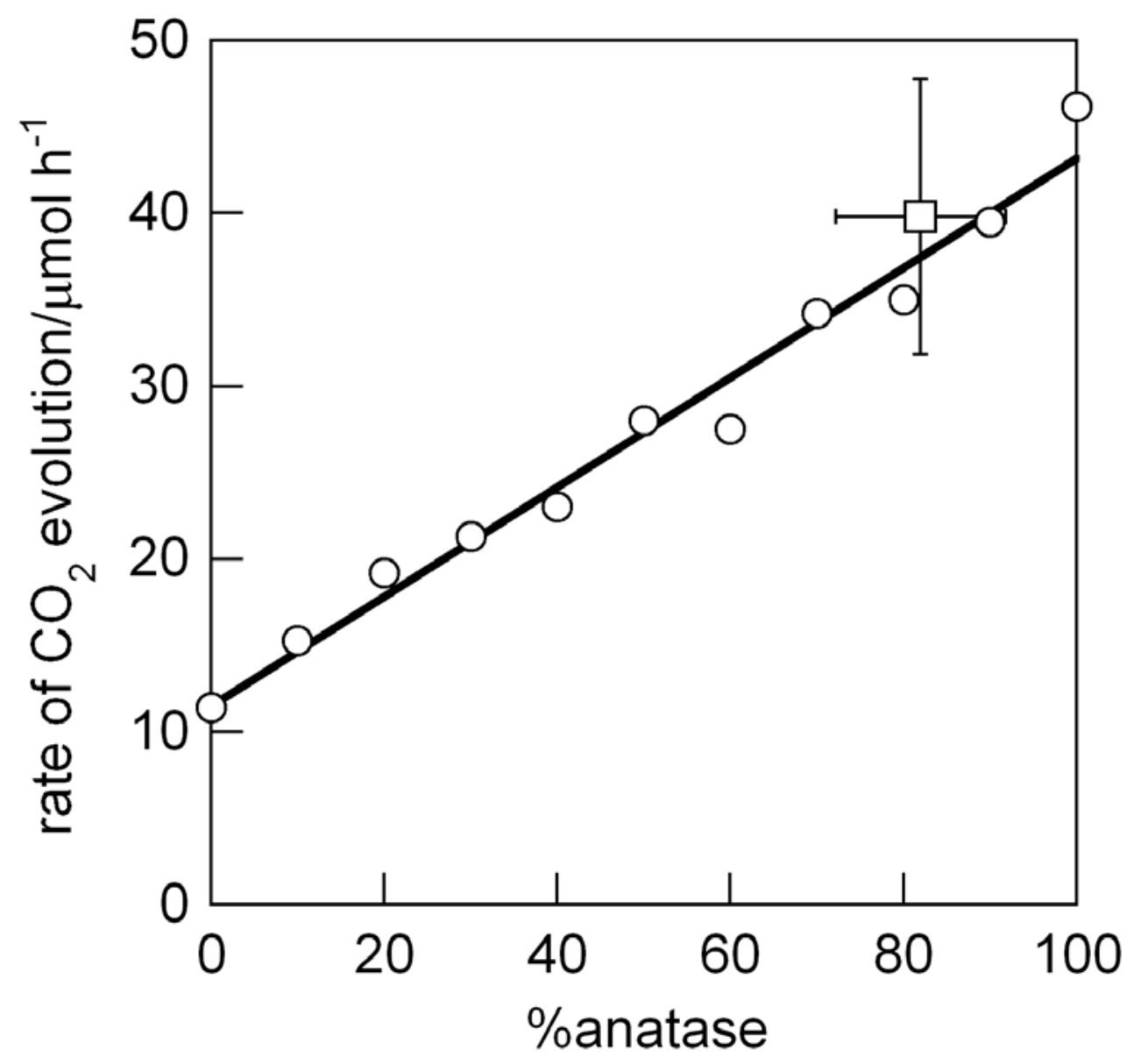

